\title{
Patient-reported outcomes in clinical trials
}

\author{
Florence Joly ${ }^{1}$, Gloria Mittica ${ }^{2}$
}

\begin{abstract}
Patient-reported outcomes (PROs) are progressively being included in clinical trials to provide information about treatment benefits identified by the patients themselves that extend the data on traditional clinical trial endpoints, such as disease free survival, overall survival, progression-free survival, and response rate. PROs may have a greater impact for patients than other endpoints. For example, patients may be prepared to forgo some increase in progression-free survival in return for reduced treatment-related toxicity. PROs may also be an indicator of disease response and have value as prognostic factors. This article discusses the way PROs can be defined and incorporated into clinical trials to enhance the value of clinical trials data and improve the understanding of the clinical benefits of a specific treatment, not only for health care professionals, but for patients and caregivers. The importance and relevance of a patient-centered perspective and shared decision making in defining value and determining treatment benefit is increasingly recognized. However, despite the acknowledged value of PROs, their inclusion in clinical trials remains far from ideal. New guidelines from the research community and technological improvements in data collection and analytics will increase the quality and the importance of PROs as standard methods for the evaluation of medical studies and in the drugs approval process.
\end{abstract}

Key words: clinical benefit, clinical trial endpoints, CONSORT PRO extension, patient-reported outcomes, reporting quality

\section{Introduction}

The most frequent endpoints of clinical trials are: disease free survival (DFS) and overall survival (OS), frequently used in the adjuvant or curative setting; progression-free survival (PFS), OS and response rate (RR), more often employed in the metastatic diseases. Nevertheless, these parameters are not completely able to reproduce the clinical benefits and risks of the therapies. Since the last decade, patient-reported outcomes (PROs) have been progressively included in clinical trials, in order to produce more complete information about the benefits of treatments as reported by the patients themselves. PROs could have a more significant impact for patients than other endpoints,

'Department of Medical Oncology, Centre François Baclesse, Caen, France.

${ }^{2}$ Department of Medical Oncology, University of Turin Medical School, Fondazione del Piemonte per l'Oncologia-Candiolo Cancer Institute (IRCCS), Candiolo (Torino), Italy.

Correspondence to: Gloria Mittica MD, Department of Medical Oncology, University of Turin Medical School, Fondazione del Piemonte per I'Oncologia-Candiolo Cancer Institute (IRCCS),

Strada Provinciale 142, Km 3,95, 10060 Candiolo (TO), Italy.

Phone: +39 0119933250 - Fax: +39 0119933275

E-mail: gloria.mittica@ircc.it

CANCER BREAKING NEWS 2016;4(2):54-58

DOI: $10.19156 / \mathrm{cbn} .2016 .0019$ as shown by Havrilesky et al. in a pilot study, which reported that women with ovarian cancer are ready to sacrifice some of meaningful endpoints, such as PFS time, for less heavy treatment-related toxicity [1]. Moreover, improvements in PROs may be correlated with disease response, as suggested in a meta-analysis conducted in 2006 [2]. In some studies, PROs have been recognized as prognostic factors $[3,4]$. The association of quality of life (QoL) parameters and symptoms (e.g. pain and weight loss) with clinical characteristics and socio-demographic variables (e.g. age, gender, performance status, stage of the disease) can increase the prediction of OS by $6 \%$, in comparison with the use of clinical characteristics and socio-demographic variables alone [3]. In a recent study presented by Roncolato et al., baseline QoL data reported that low physical function, role function, global health status and high abdominal or gastrointestinal symptoms are predictors for OS and for stopping chemotherapy early in platinum-resistant/refractory ovarian cancer [5].

\section{Definitions and measures}

PROs are defined as any report of the status of a patient's health condition, originated from the patient himself, without interpretation of the patient's response by the clinician or anyone else [6]. PROs can include different aspects 
of the health condition, such as QoL, health related QoL (HRQoL), patient's satisfaction with care, symptoms, pain, psychological distress, self experience and patientreported adherence to therapy. These data are collected in most of cases by the patients directly through standardized and validated questionnaires, such as the European Organization for Research and Treatment of Cancer (EORTC) QoL questionnaires or Functional Assessment of Cancer Therapy (FACT) questionnaires. A possible alternative is to collect the spontaneous feedback of patients, with the help of caregivers, especially in the elderly population and/ or in the palliative setting, when patients are not independent [6].

Others measures have been developed to assess the value of therapies, including the widely used metric of qualityadjusted life-years (QALYs). QALYs are an indicator of disease burden, that includes both quality and quantity of life lived. The QALYs measure, collected on a selected population of patients, provides an indication about the benefits obtained from medical procedures in terms of QoL and survival. QALYs is used to evaluate and compare the effectiveness of different treatments, enabling the choice of the most cost-effective one. QALYs can be used as parameter for decision making for specific patients, but acquires more importance when is used for statistical and economical evaluations $[7,8]$. Since published studies have demonstrated an under-reporting of patients' symptoms and their agreement between the evaluations of patients and clinicians, the Patient Reporting of Common Terminology Criteria for Adverse Events (PRO-CTCAE) library has been developed $[6,9,10]$. It includes 78 symptomatic side effects and adverse events (AEs) commonly reported in oncology clinical trials and assesses their frequency, severity and interference with daily activities. The patients' AEs reports could give additional information particularly in the target therapy era, when drugs should be taken for an extended time, in order to more deeply analyze the overall benefit [10].

Composite endpoints including PROs, tumor response and survival outcomes have been developed in order to enhance the value of clinical trial results and to assist the deeper understanding of the entire clinical benefits of a specific treatment, both by health care professionals (HCP), patients and caregivers [4]. Different composite endpoints for PROs have been proposed, such as clinical benefit response (CBR) endpoint, time until definitive deterioration (TUDD) and overall treatment utility (OTU) [4, 11-13]. In this context, CBR endpoint includes pain (result of both analgesic use and patient-reported pain intensity scale), clinician-recorded performance status and weight. CBR was initially defined by Burris et al. and, subsequently, widely used across the literature [11]. CBR should be not confused with the definition of clinical benefit reported in tumor-centered trials, where it indicates the changes of tumor size after treatments (complete response, partial response and stable disease). TUDD is calculated in different ways according to the studies in which it is used. Bonnetain et al. evaluated QoL every 8 weeks until death through these parameters: global health, emotional functioning, physical functioning, fatigue and pain. Bonnetain's TUDD was calculated as the elapsed time between the start of the study and the first definitive decrease in QoL parameters (mentioned above) by 5 or more points [12]. OTU incorporates tumor response, serious adverse events, grade $>3$ non-hematological toxicity and death [13]. However, there is not a consensus about the treatment related symptoms in composite endpoints, which are not yet widely validated and used.

The relevance of patient centeredness and of patient perspective in defining value has been recognized by the American Society of Clinical Oncology (ASCO) and the European Society for Medical Oncology (ESMO). In order to help HCP and patients to share decision making, ASCO developed a value framework, divided into two different versions, one for curative settings and one for advanced disease. Both models consider clinical benefits (including the most frequently used endpoints, OS, PFS and RR) and toxicity (this value represents the delta between the toxicity of the new agent and the comparator therapy). For advanced disease, the model includes a PROs endpoint, as "bonus point", recognizing improvements in cancerrelated symptoms and prolongation of the treatment-free interval. The prolongation of treatment-free interval is supposed to be a good-health substitute, since it indicates a vacation from drug toxicities [7].

ESMO has also recently developed a Medical Oncology Magnitude of Clinical Benefit Scale (ESMO-MCBS), in order to evaluate the meaningful benefits obtained from a new treatment. The ESMO-MCBS incorporates OS, PFS and QoL. In particular, QoL can upgrade or downgrade the evaluation of the new drug, respectively when a statistically significant improvement in QoL or its delayed deterioration have been recorded or, on the contrary, when an absence of QoL advantage has been reported [14].

\section{How to include PROs in clinical trials: the CONSORT PRO extension}

Although PROs are important endpoints in clinical decision making, and even if PRO measures are included in studies, only few randomized controlled trials (RCTs) adequately report PRO data. In a review published in 2011, Brundage et al. reported that in an identified sample of 794 RCTs, 
PROs were primary endpoints in just $26 \%$ of the total trials; in the $56 \%$ of the total trials where the rationale for the selected PROs was documented, $28 \%$ of the RTCs gave information about missing data and $64 \%$ of the RTCs analyzed the PRO results in the context of other endpoints [15]. The sub-optimal protocols for reporting PROs in clinical trials are also confirmed by two recent articles focused, respectively, on ovarian cancer and advanced breast cancer, in particular regarding timing of administration of PROs instruments, monitoring of PROs compliance, handling of missing data, the analysis plan for PROs and results discussion $[16,17]$.

Since the interpretation of PROs data requires accurate and standardized reporting, the Consolidated Standards of Reporting Trials (CONSORT) Statement developed a PROs centered extension. The CONSORT PRO guidance identified 5 objectives that must be present in all RCTs in which PROs are primary or significant secondary endpoints:

1. PROs should be recognized as primary or secondary endpoints in the abstract;

2. if a multidimensional PROs instrument is used, the hypothesis and relevant fields must be described;

3. the evidence of the PROs tool's validity (evaluate what they are designed to measure) and reliability (get the same answer repeatedly) should be explained or mentioned;

4. the statistical approaches to manage the missing data should be clearly explained, since missing data reduce power of the study and is a potential cause of bias;

5. PRO-specific restrictions of study results and generalizability of findings to other populations and clinical practice should be discussed.

In the results section, baseline PROs data must be presented, since they could be useful to assess the importance of trial findings. If possible, additional PROs or the elements of composite PRO scores should be analyzed in the main publication (or in a companion second publication), in order to reduce selection bias of reporting only significant results. In fact, some studies do not take into account the clinical relevance of PROs, especially in RCTs where there are discrepancies between PROs themselves and survival endpoints [18].

\section{When and how PROs could be included in clinical trials}

In advanced disease, the objective is to improve survival without degradation of QoL. For this reason, the inclusion of PROs assumes a particular relevance, more particularly when the first goal is to improve palliation of symptoms. Symptoms control or improvement, with the consequent advantage in HRQoL, represents a more important and substantial clinical benefit than RR (according to Response Evaluation Criteria In Solid Tumors [RECIST] criteria) or PFS alone. Collection of HRQoL is important not only during the treatment, but also after and during subsequent lines. Data might evidence a delay in the onset of cancerrelated symptoms and a postponement of following treatments. The extension of HRQoL data recording after the end of therapy could evidence late onset toxicities and related symptoms. In advanced disease, even if the treatment is becoming similar to other chronic diseases like hypertension or epilepsy, the approval of new drugs is exceptionally based only on reduction of symptoms [19]. Even if the Food and Drug Administration (FDA)'s Guidance to Industry in 2007 suggested that symptom endpoints can be sufficient in the regular drugs approval process, in practice only mitoxantrone in metastatic prostate cancer has been approved based on symptoms palliation [20, 21].

A patient-centered outcome research, based on standardized PROs, can also add important value in adjuvant therapy and in asymptomatic relapse. In the adjuvant setting, assessing acute and long term side-effects of the treatment is an important goal. In some cases, for a marginally improvement in survival, these patients could decide to refuse the proposed treatment or to postpone it at the onset of symptoms. In this setting the collection of HRQoL data with reference to late onset toxicities acquires more importance, such as anthracycline related heart impairment and peripheral neuropathy, that could significantly worsen daily activities.

In the area of targeted therapies and immune checkpoint inhibitors prescribed for long periods and sometimes in the maintenance setting, the evaluation of PROs, in particular the ones related to chronic toxicities and to adherence of therapies, will continue to become more important. Many of the targeted therapies are small molecules with an oral administration and the patient's compliance assumes a fundamental role for their efficacy [6]. The new agents have different toxicity profile in comparison to chemotherapy and the value of using RECIST criteria to assess response is under review. In this setting, inclusion of PROs data as endpoints can help in understanding trial results and in evaluating the overall benefit. For example, the proportion of patients that reached a significant improvement in symptoms from baseline at a predefined time point is perhaps more important than a modest rate of disease progression based on RECIST criteria or a marginal PFS difference of some weeks [22].

\section{Future perspectives and conclusion}

The importance of including PROs in clinical trials is largely recognized today, according to the CONSORT PRO ex- 
tension recommendations. Nevertheless, a recent review by Bylicki et al. found that $62 \%$ of RCTs did not report PROs, while the other ones lacked information about items associated with methods of PROs collection and analysis (just $16 \%$ of the RTCs recorded methods for data collection and only $37 \%$ described the management of missing data) [23]. The presence of a dedicated secondary manuscript was an independent predictor of overall quality score [23]. Starting from 2007, the FDA's Guidance to Industries included symptoms as important endpoint for regular drug approval. For this reason, it is important to encourage the inclusion of PROs in the endpoints of RCTs. The new technologies, such as the digital conversion of the traditional paper questionnaires and the big data analytics, will increase the capture and statistical analyses of PROs data, with a more strict observation of the pre-scheduled detection and a reduction of the amount of missing data. A positive trend for reduction of missing data and an increment in compliance rate

\section{References}

1. Havrilesky LJ, Alvarez Secord A, Ehrisman JA et al. Relative influence of factors determining a woman's preference for treatment options in ovarian cancer. Proc Am Soc Clin Oncol 2014;32(abstr 5544).

2. Victorson D, Soni M, Cella, D. Metaanalysis of the correlation between radiographic tumor response and patientreported outcomes. Cancer 2006;106(3):495-504.

3. Quinten C, Coens C, Mauer M et al. Baseline quality of life as a prognostic indicator of survival: a meta-analysis of individual patient data from EORTC clinical trials. Lancet Oncol 2009;10(9):865-71.

4. Secord AA, Coleman RL, Havrilesky LJ et al. Patient-reported outcomes as end points and outcome indicators in solid tumors. Nat Rev Clin Oncol 2015;12(6):358-70.

5. Roncolato F, O'Connel R, Buizen L et al. Baseline quality of life (QOL) as a predictor of stopping chemotherapy early, and of overall survival, in platinum-resistant/ refractory ovarian cancer (PRROC): The GCIG symptom benefit study (SBS) (abstract). J Clin Oncol 2016;34(15 suppl):Abstr 5508.

6. Banerjee AK, Okun S, Edwards IR et al. Patient-reported outcome measures in safety event reporting: PROSPER Consortium guidance. Drug Saf 2013;36(12):1129-49.

7. Schnipper LE, Davidson NE, Wollins DS et al. American Society of Clinical Oncology Statement: a conceptual framework to assess the value of cancer treatment options. J Clin Oncol 2015;33(23):2563-77.

8. Weinstein M, Torrance G, Mc Guire A. QALYs: the basics. Value Health 2009;12(suppl 1):S5-9.

9. Strong LE. The past, present, and future of patient-reported outcomes in oncology. Am Soc Clin Oncol Educ Book 2015:e616-20.

10. Kluetz PG, Chingos DT, Basch EM et al. Patient-reported outcomes in cancer clinical trials: measuring symptomatic adverse events with the National Cancer Institute's Patient- (between 84.7 and 97.2\%) have been reported recently in Alliance and Mayo Clinic trials [24].

The research community guidelines, developed during the past years based on several different studies, and the technological improvements about data collection and analytics will increase the quality and the importance of PROs as standard methods for the evaluation of medical studies and in the approval processes for new drugs.

\section{Acknowledgments}

The authors thank Ray Hill, an independent medical writer, who provided native English editing and journal styling on behalf of HPS. This editorial assistance was funded by PharmaMar, Spain.

\section{Conflicts of Interest}

The Authors declare there are no conflicts of interest in relation to this article.

Reported Outcomes Version of the Common Terminology Criteria for Adverse Events (PRO-CTCAE). Am Soc Clin Oncol Educ Book 2016;35:67-73.

11. Burris HA, Moore MJ, Andersen J et al. Improvements in survival and clinical benefit with gemcitabine as first-line therapy for patients with advanced pancreas cancer: a randomized trial. J Clin Oncol 1997;15(6):2403-13.

12. Bonnetain F, Dahan L, Maillard E et al. Time until definitive quality of life score deterioration as a means of a longitudinal analysis for treatment trials in patients with metastatic pancreatic adenocarcinoma. Eur $\mathrm{J}$ Cancer 2010;46(15):2753-62.

13. Seymour MT, Thompson LC, Wasan HS et al. Chemotherapy options in elderly and frail patients with metastatic colorectal cancer (MRC FOCUS2): an open-label, randomised factorial trial. Lancet 2011;377(9779): 1749-59.

14. Cherny NI, Sullivan R, Dafni U et al. A standardized, generic, validated approach to stratify the magnitude of clinical benefit that can be anticipated from anti-cancer therapies: the European Society for Medical Oncology Magnitude of Clinical Benefit Scale (ESMO-MCBS). Ann Oncol 2015;26(8):1547-73.

15. Brundage M, Bass B, Davidson J et al. Patterns of reporting health-related quality of life outcomes in randomized clinical trials: implications for clinicians and quality of life researchers. Qual Life Res 2011;20(5):653-64.

16. Mercieca-Bebber R, Friedlander M, Kok PS et al. The patient-reported outcome content of international ovarian cancer randomised controlled trial protocols. Qual Life Res 2016 Jun 13. [Epub ahead of print].

17. Turner-Bowker DM, Hao Y, Foley C et al. The use of patient-reported outcomes in advanced breast cancer clinical trials: a review of the published literature. Curr Med Res Opin 2016 Jun 22:1-37. 
18. Calvert M, Blazeby J, Altman DG et al. Reporting of patient-reported outcomes in randomized trials: the CONSORT PRO extension. JAMA 2013;309(8):814-22.

19. Wilson MK, Collyar D, Chingos DT et al. Outcomes and endpoints in cancer trials: bridging the divide. Lancet Oncol 2015;16(1):e43-52.

20. U.S. Department of Health and Human Services, Food and Drug Administration. Guidance for industry: Clinical trial endpoints for the approval of cancer drugs and biologics 2007. Available from: www.fda.gov/downloads/Drugs/ Guidances/ucm071590.pdf.

21. Tannock JF, Osoba D, Stockler MR et al. Chemotherapy with mitoxantrone plus prednisone or prednisone alone for symptomatic hormone-resistant prostate cancer: a Canadian randomized trial with palliative end points. J Clin Oncol 1996;14(6):1756-64.

22. Friedlander M, Mercieca-Bebber RL, King MT. Patient-reported outcomes (PRO) in ovarian cancer clinical trials-lost opportunities and lessons learned. Ann Oncol 2016;27(suppl 1):i66-71.

23. Bylicki O, Gan HK, Joly F et al. Poor patient-reported outcomes reporting according to CONSORT guidelines in randomized clinical trials evaluating systemic cancer therapy. Ann Oncol 2015;26(1):231-7.

24. Atherton PJ, Burger KN, Pederson LD et al. Patient-reported outcomes questionnaire compliance in Cancer Cooperative Group Trials (Alliance N0992). Clin Trials 2016 Jun 30. [Epub ahead of print]. 